\title{
Obstetric Study of Second Pregnancy after Cesarean Section
}

\author{
Lili Xuan", Xiaojun Liu*,1 \\ ${ }^{1}$ China-Japan Union hospital of Jilin University, Changchun, Jilin, 130033 \\ *corresponding author
}

Keywords: Second Pregnancy; Cesarean Section; Obstetric Study

\begin{abstract}
With an increase in the number of cesarean sections, there is an increased risk of serious complications in re-pregnancies. After cesarean section, the second pregnancy is too short and the risk of serious complications increases. However, due to the inconsistent conclusions of relevant studies, the optimal timing for re-pregnancy after cesarean section is not yet clear, and can only be based on the existing evidence to comprehensively determine the level of risk, and for clinical guidance.
\end{abstract}

\section{Introduction}

Due to a variety of reasons, the cesarean section rate in the country has increased year by year, reaching 50\%, and in some areas it has even reached more than $60 \%$. Caesarean section may increase the risk of complications in the next pregnancy, such as uterine rupture, placenta previa, placental adhesions, and bleeding-related complications; and with the increase in the number of cesarean section and the short interval between two gestations, will further increase the risk of mother and child [1]. At present, China is in the period of the first generation child with only childbearing peak and excessive cesarean section rate. It is particularly important to determine the effect of different intervals of pregnancy and the number of cesarean sections on the adverse outcomes of mother and child pregnancy. The risk of second pregnancy after cesarean section is related to the risk of uterine rupture, placenta previa, placental adhesion, and postpartum hemorrhage. Therefore, the risk of re-pregnancy after cesarean section assessment is mainly to assess the risk factors. In brief, it is mainly the assessment of the effect of the interval between two pregnancies and the number of cesarean deliveries on pregnancy.

\section{Factors Affecting Uterine Incision Repair}

The condition of the previous cesarean section affected the repair of the uterine incision, which in turn affected the outcomes of the re-pregnant mother and child, such as whether the cesarean section of the previous cesarean section, whether the cesarean section had labor, the indication of cesarean section, and the uterus. Incision type, suture method of uterine incision, and recovery after cesarean section.

The most commonly used incision in cesarean section is a transverse incision in the lower uterus, and there are classical incisions, $\mathrm{T}$ incisions, and straight incisions in the lower uterus. Classical incision and T-shaped incision are the most unfavorable for the healing of uterine incisions. The incidence of uterine rupture during re-pregnancy is $4 \%$ to $9 \%$, which can occur before full-term pregnancy, before labor or during labor, and the transverse incision of the lower uterus. The rate of rupture during pregnancy is $0.2 \%$ to $0.9 \%$.

The suture of the uterine incision (single or bilateral, seaming) The uterine incision suture for caesarean section does not affect the rupture of the uterus during the next pregnancy (especially during vaginal trial production after caesarean section). Clear. Therefore, different hospital doctors in different hospitals and even the same hospital do not have the same uterine suturing methods. Some scholars believe that the uterine incision suture method (single or double layer) has no effect on the rupture of the uterus in the next pregnancy, but the sample size of the above study is relatively small (292 cases and 145 cases). However, some scholars have reported that although 
there is no difference in the incidence of uterine rupture, single-layer suture increases the risk of uterine dehiscence during second pregnancy. Other scholars reported that compared with double sutures, the risk of uterine rupture after single-skin suturing increased nearly 4 times (OR 3.95, 95\% CI 1.35 to 11.49), and it was reported that once again after single suture The prevalence of uterine rupture in pregnancy increased by 8 times (OR 8.01, 95\% CI 1.96 to 32.79), so it is presumed that it may be related to the following factors: Single-layer suturing does not make the aponeurosis and aponeurosis, muscularis and muscular layer in good alignment, And the second layer of suture can be further on the myometrium. Although single-layer sutures can reduce operative time and reduce the incidence of postpartum endometritis (13.5\% vs. $25.5 \%)$, it is not fully understood whether the effect of monolayer or bilateral sutures on uterine rupture during re-pregnancy is currently unclear, and our country The first-generation only child is in the reproductive period. It is necessary to carefully consider whether to adopt a single-layer suture in the first cesarean section. Multi-center randomized controlled trials are required to further elucidate what is excellent.

Whether the gestational age at the previous cesarean section was related to the risk of rupture of the uterus during the second pregnancy was performed by the National Institute for Child Health and Human Development (NICHD) Maternal-Fetal Medicine Unite Networks (MFMU) from 1999 to 2001. In the study on the risk of recurrent pregnancy in the history of cesarean section, if the previous cesarean section was not full-term, the risk of rupture of the uterus during the second pregnancy was increased compared with the previous full-term cesarean section $(0.58 \% \mathrm{vs} .0 .28 \%$, $\mathrm{P}<0.001$ ); If the elective cesarean section is excluded, the risk of uterine rupture is still increased $(0.79 \%$ vs. $0.46 \%, P=0.001)$ during the previous pregnancy with cesarean delivery for the first time; the multivariate analysis after controlling for confounding factors shows that The risk of uterine rupture was increased by 1.6 times (OR 1.6, 95\% CI 1.01 to 2.5) during the second pregnancy without previous full-term cesarean delivery [2]. Therefore, it is speculated that may be related to the following factors: premature cesarean section when the formation of the lower uterine segment is relatively poor, may involve the uterine body during cesarean section; premature cesarean section and subclinical infection, premature rupture of membranes, etc., local cytokine may It can affect the repair of postoperative uterine incisions, thereby increasing the risk of uterine rupture during a second pregnancy.

\section{Effects of Different Intervals of Pregnancy on Pregnancy Outcomes}

The effect of pregnancy interval on the outcome of pregnancy includes two meanings. The first is the effect of pregnancy interval (regardless of the previous delivery method) on the outcome of mother and child. If the short pregnancy interval causes the next pregnancy, low birth weight, premature delivery, premature delivery Premature rupture and increased incidence of placenta previa; second is the effect of a second gestational interval after cesarean section on uterine incision repair, ie uterine rupture and its associated maternal and child risks (this article focuses on the second point). The repair of uterine smooth muscle is very slow. It takes a long time to repair the uterine incision smooth muscle after cesarean section. MRI and hysteroscopy revealed that the scar repair of the uterine incision is not complete after 6 to 12 months, so it is inferred that: Short re-pregnancy after the birth of the uterus will make the uterine incision smooth muscle not have enough time to repair, so that the risk of maternal and child increase in the next pregnancy (especially the risk associated with uterine rupture, such as: fetal death, severe neonatal ischemia and hypoxia Encephalopathy, maternal death, etc.).

\section{Effect of Different Cesarean Delivery Times on Pregnancy Outcomes}

With the increase in the number of cesarean sections, there is an increased risk of serious complications of maternal complications associated with uterine rupture, placenta previa, placental adhesions, and hysterectomy. The relationship between the number of cesarean sections and rupture of the uterus (especially the risk of rupture of the uterus during trial production) is not yet clear. Most scholars believe that as the number of cesarean sections increases, the risk of uterine rupture 
increases. Studies have investigated the production of 12707 pregnant women with a history of cesarean section. The risk of uterine rupture was $0.6 \%$ in the history of 1 cesarean section, and the risk of uterine rupture was $1.8 \%$ in 2 or more cesarean birth history groups (OR 3.06, 95\%CI1.95 to 4.79), but this study did not provide further analysis of the drug induced or induced by labor during trial production (how much is the incidence of uterine rupture during oxytocin and prostaglandin induction or oxytocin production?). The study also reported that after controlling for prostaglandins, oxytocin, and other confounding factors, the incidence of uterine rupture in pregnant women with a history of 2 cesarean sections was increased by 4.8 times during trial production compared with women with a history of cesarean section (OR4). .8, 95\% CI1.8 to 13.2). However, in 2006, there were studies comparing the proportion of 975 cases of cesarean section with 2 cesarean sections and 16 cases of 1 cesarean section with uterine rupture at trial production using the MFMU network database, which were $0.9 \%$ and $0.7 \%$, respectively. The difference was not statistically significant. And scholars in Saudi Arabia reported that even if 3 or more cesarean sections do not increase the risk of rupture of the uterus, this is not in line with our traditional understanding: With the increase in the number of cesarean sections, the uterine scar becomes more and more fragile, which makes it easier Uterine rupture occurred. At present, the American College of Obstetricians and Gynecologists (ACOG) (2004) does not support vaginal trial production for pregnant women with 2 or more cesarean section history [6], while the Canadian Association of Obstetricians and Gynecologists (SOGC) (2005) Women who did not explicitly oppose 2 or more cesarean sections had vaginal trials, but they also noted a significant increase in the risk of uterine rupture. The concept of "one cesarean section and cesarean section forever" has gradually changed in the past 30 years. Vaginal trial production can be performed after cesarean section. Interestingly, many studies have found that if vaginal delivery is successfully performed after cesarean section, the risk of uterine rupture and other complications will be reduced, and the risk of uterine rupture will decrease as the number of vaginal delivery increases. Some scholars have reported that after cesarean section, if there are $0,1,2$ and 3 successful vaginal delivery experiences, the incidence of uterine rupture is $1.6 \%, 0.3 \%, 0.3 \%$ and $0.35 \%$ respectively. Although the risk of uterine rupture is extremely high, the absolute number of occurrence is still relatively low; and placenta and placental adhesions associated with the number of cesarean sections are more common, and can cause severe bleeding and hysterectomy during delivery. Should pay attention to. In 1954, Bender first reported the relationship between the history of cesarean section and placenta previa. In 1997, Ananth et al. conducted a meta-analysis of cesarean section and placenta previa: 1 history of cesarean section, placenta previa. The relative risk (RR) value was 4.5 (95\% CI 3.6 to 5.5); the history of 2 cesarean sections was RR 7.4 (95\% CI 7.1 to 7.7); the history of 3 cesarean sections was RR 6.5 (95\% CI 3.6 to 11.6); 4 times history of cesarean section, RR 44.9 (95\% CI 13.5 to 149.5). Similarly, as the number of cesarean sections increases, the risk of placental adhesions increases. If the history of 1 cesarean section was used as a control, the risk of placental adhesions increased by 1.3 times for women who had 2 or 3 times, 4 times, 5 times, and 6 or more cesarean sections (OR 1.3, 95). \%CI0.7 to 2.3), 2.4 times (OR2.4, 95\%CI1.3 to 4.3), 9.0 times (OR 9.0, 95\% CI4.8 to 16.7), 9.8 times (OR 9.8, 95\% CI3.8 25.5) and 29.8 times (OR29.8, 95\% CI 11.3 to 78.7). Similarly, if the history of 1 cesarean section was used as a control, the risk of hysterectomies was increased by 1.4 times in women with a history of cesarean section at 3, 4, 5, and 6 times or more and 6 times (OR 1.4, 95\% CI $0.9 \sim 2.1$ ), 3.8 times (OR 3.8, 95\% CI2.4 to 6.04), 5.6 times (OR 5.6, 95\% CI2.7 to 11.6) and 15.2 times (OR 15.2, 95\% CI6.9 to 33.5). For placenta previa, the incidence of placental adhesions will increase with the increase in the number of cesarean sections: the incidence of placental adhesions in the history of 1 cesarean section will be $25 \%$, the incidence of placental adhesions in 2 cesarean sections will be $>40 \%$, and 3 History of cesarean section incidence of placental adhesions $>60 \%$

\section{Conclusion}

There are many factors related to the safety of re-pregnancy after cesarean section, such as 
vaginal bleeding after the previous cesarean section, whether breast-feeding, whether the production of rickets rate and the detection of the thickness of the lower uterine segment during pregnancy after pregnancy, pregnant women in late pregnancy The symptoms and signs of precursor rupture of the uterus, the threatened labor at the hand, and the thickness of the lower uterine segment measured at different times after labor, the stress that the uterine scar can withstand, and the safety of the vaginal delivery will be of interest to the obstetrician. .

\section{References}

[1] Li Xiuyan, Wang Changkai. Clinical analysis of incomplete rupture of uterine scars [J]. Journal of Liaoning Medical University, 2010, 131 (6): 498-499,513

[2] He Hongyu. Late pregnancy scar incomplete rupture of 18 cases of clinical analysis [J]. Chinese Modern Drug Application, 2011, 5(14): 93-94

[3] Zhu Xi. The length of secondary cesarean section on the outcome of pregnancy [J]. Practical Integrated Traditional Chinese and Western Medicine, 2013, 13(2): 66-67

[4] Chen Yisong, Hua Keqin. Short-term uterine scar pregnancy safety analysis [J]. Advances in Modern Obstetrics and Gynecology, 2009, 18(9): 648-651

[5] Society of Obstetricians and Gynecologists of Canada. Guidelines for vaginal birth after previous cesarean birth[J]. Int J Gynaecol Obstet, 2005, 89(3): 319-331

[6] Xiong Hao, Li Xiaotian. Common causes of scar uterus and its impact on long-term pregnancy [J]. Chinese Journal of Practical Gynecology and Obstetrics 2010,26(8):577-579 